\title{
Presenilins as endoplasmic reticulum calcium leak channels and Alzheimer's disease pathogenesis
}

\author{
Charlene SUPNET \& Ilya BEZPROZVANNY* \\ Department of Physiology, University of Texas Southwestern Medical Center at Dallas, Dallas TX 75390-9040, USA
}

Received May 18, 2011; accepted June 08, 2011

\begin{abstract}
Alzheimer disease (AD) is the most common neurodegenerative disorder worldwide and is at present, incurable. The accumulation of toxic amyloid-beta $(\mathrm{A} \beta)$ peptide aggregates in $\mathrm{AD}$ brain is thought to trigger the extensive synaptic loss and neurodegeneration linked to cognitive decline, an idea that underlies the 'amyloid hypothesis' of AD etiology in both the familal (FAD) and sporadic forms of the disease. Genetic mutations causing FAD also result in the dysregulation of neuronal calcium $\left(\mathrm{Ca}^{2+}\right)$ handling and may contribute to AD pathogenesis, an idea termed the 'calcium hypothesis' of AD. Mutations in presenilin proteins account for majority of FAD cases. Presenilins function as catalytic subunit of $\gamma$-secretase involved in generation of $A \beta$ peptide Recently, we discovered that presenilns function as low-conductance, passive ER $\mathrm{Ca}^{2+}$ leak channels, independent of $\gamma$-secretase activity. We further discovered that many FAD mutations in presenilins result in loss of ER $\mathrm{Ca}^{2+}$ leak function activity and $\mathrm{Ca}^{2+}$ overload in the ER. These results provided potential explanation for abnormal $\mathrm{Ca}^{2+}$ signaling observed in FAD cells with mutations in presenilns. Our latest work on studies of $\mathrm{ER} \mathrm{Ca}^{2+}$ leak channel function of presenilins and implications of these findings for understanding $\mathrm{AD}$ pathogenesis are discussed in this article.
\end{abstract}

calcium signaling, neurodegeneration, presenilins, Alzheimer's disease, gamma-secretase, amyloid

Citation: Supnet C, Bezprozvanny I. Presenilins as endoplasmic reticulum calcium leak channels and Alzheimer's disease pathogenesis. Sci China Life Sci, 2011, 54: 744-751, doi: 10.1007/s11427-011-4201-y

\section{Alzheimer's disease}

Alzheimer's disease (AD) is the most common type of age-related dementia. It is a worldwide epidemic which currently affects approximately 27 million people and because there is no known cure or therapy that significantly slows disease progression, this number is projected to quadruple by 2050 [1]. Clinical characteristics of AD include a decline in memory, particularly in short-term and working memory, apathy, depression, impaired judgement, disorientation, confusion, changes in behaviour and difficulty speaking, swallowing and walking. The key pathological hallmarks of $\mathrm{AD}$ are extensive neurodegeneration of the

*Corresponding author (email: ilya.bezprozvanny@utsouthwestern.edu) median temporal lobe (the hippocampus in particular), parietal lobe, select regions of the frontal cortex and cingulate gyrus [2,3]; the presence of extracellular senile plaques composed of dense-core deposits of amyloid $\beta$ peptide $(\mathrm{A} \beta)$, dystrophic neuritis and activate microglia [4,5]; and intracellular neurofibrillary tangles (NFTs) comprised of hyperphosphorylated microtubule-associated protein tau (MAPT) [6]. The majority of $\mathrm{AD}$ cases are sporadic (SAD) and characterized by the late age of disease onset ( $>60$ years of age). A small percentage $(1 \%-2 \%)$ of all $\mathrm{AD}$ cases are characterized by an earlier onset ( $<60$ years) and genetic inheritance, known as familial AD (FAD). Mutations in the genes encoding presenilin-1 (PSEN1), presenilin-2 (PSEN2) and amyloid precursor protein $(A P P)$ account for more than $50 \%$ of autosomal-dominant FAD cases $[4,5,7,8]$. Mutations in the MAPT gene do not lead to AD but instead result in a 
variant of frontotemporal dementia (FTDP17) $[6,9,10]$.

\section{Presenilins and familial Alzheimer's disease}

The majority of FAD is caused by missense mutations in the PSEN1 and PSEN2 genes, which display $80 \%$ sequence homology. Since the discovery of their potential involvement in AD pathogenesis over 16 years ago [11], 182 mutations in PSEN1 and 14 in PSEN2 have been identified to be causative in FAD (www.molgen.ua.ac.be/ADMutations) $[12,13]$. Presenilins (PSs) belong to the family of aspartic proteases and they are involved in Regulated Intramembrane Proteolysis (RIP), a mechanism that is used to cleave peptide bonds within the lipid bilayer $[14,15]$ (reviewed in [16]). Presenilins are $50 \mathrm{kD}$ holoproteins that contain 9 transmembrane (TM) domains $[17,18]$ and they reside primarily in the endoplasmic reticulum (ER) membrane [19] with the amino-terminal oriented towards the cytosol and the carboxy-terminal toward the ER lumen (Figure 1). The PSs aggregate with nicastrin, anterior pharynx defective 1 (Aph-1) and presenilins enhancer 2 (Pen-2) subunits to produce the multimeric $\gamma$-secretase complex, which is transported to the cell surface and endosomal structures to cleave type I transmembrane proteins such as Notch and the amyloid precursor protein (APP). Following assembly, PS1 and PS2 holoproteins undergo endoproteolysis in the cytosolic loop between TM6 and TM7, resulting in the generation of a $35 \mathrm{kD}$ amino-terminal fragment (PS-NTF) and an 18-20 kD carboxy-terminal fragment (PS-CTF), which remain associated with each other in the "mature" $\gamma$-secretase complex [20-27]. The cleavage of APP sequentially by $\beta$-secretase then $\gamma$-secretase results in the generation and release of $A \beta$, the principal constituent of the amyloid $\beta$ in the brains of AD patients [27,28]. Consistent with the role of PSs as the catalytic subunits of $\gamma$-secretase $[8,27,28]$, PS-FAD mutations result in a shift in the proteolysis of APP such that there is an increase in the production the hydrophobic, aggregation-prone 1-42 A $\beta$ fragment, increasing $\mathrm{A} \beta 42 / 40$ ratio and contributing to $\mathrm{A} \beta$ plaque formation [27-29]. In addition, loss of PSs or mutation of the catalytic aspartates D257 or D385 in TM6 and TM7, respectively, affect APP processing (Figure 1) [27].

\section{Presenilins and intracellular calcium signaling}

The identification of the biological functions of PS has been a global effort since the discovery that they were involved in AD pathogenesis. It appears that PSs have a wide range of functions beyond RIP and $\gamma$-secretase function, including $\beta$-catenin regulation, protein trafficking, apoptosis in both mammals and plants (reviewed in [16]). Interestingly, many PS-FAD mutations result in deranged neuronal calcium $\left(\mathrm{Ca}^{2+}\right)$ signaling (reviewed in [30-33]), which suggests a role for PS in the regulation of intracellular $\mathrm{Ca}^{2+}$ signaling. The connection between PS-FAD mutations and abnormal $\mathrm{Ca}^{2+}$ signaling has been known approximately 2 decades [34], however, the molecular mechanism(s) behind this observation are still controversial [30-33].

A consistent observation is that PS-FAD mutations predominantly result in the exaggerated release of $\mathrm{Ca}^{2+}$ from overloaded ER stores. Skin fibroblasts from human patients that harbour a mutation in PS1-A246E showed exaggerated $\mathrm{Ca}^{2+}$ release from $\mathrm{IP}_{3}$-gated stores compared to controls after treatment with bombesin and bradykinin [34]. Alterations in $\mathrm{Ca}^{2+}$ signalling were detected before the development of overt clinical symptoms and such changes were not present in cells from subjects that failed to develop AD [35]. These initial results were recapitulated experimentally in various model systems expressing FAD-related mutations in PS and the data suggested that in addition to contributing to altered $\gamma$-secretase function, PS mutations had a significant impact on $\mathrm{Ca}^{2+}$ signalling in AD models. These early observations supported the " $\mathrm{Ca}^{2+}$ hypothesis of AD" which states that deranged $\mathrm{Ca}^{2+}$ signaling plays an important role in $\mathrm{AD}$ pathogenesis [30-33,36,37].

More recently, primary cortical neurons from mice expressing mutant PS1 displayed similar altered $\mathrm{IP}_{3} \mathrm{R} \mathrm{Ca}{ }^{2+}$ signalling [38] which were attributed to increased ryanodine receptor (RyanR) expression and recruitment [39,40]. In addition, RyanR protein levels and channel function are increased in mouse models of containing PS mutations PS1-M146V and PS2-N141I [41-43]. Clinical mutations of PS2 also enhanced $\mathrm{Ca}^{2+}$ release from $\mathrm{IP}_{3} \mathrm{R}$-gated ER stores [44]. PS1 mutations and genetic knockout attenuate capacitative $\mathrm{Ca}^{2+}$ entry (CCE), a refilling mechanism for depleted ER $\mathrm{Ca}^{2+}$ stores [45-51]. The PS1-M146V mutation augmented $\mathrm{Ca}^{2+}$ release from $\mathrm{IP}_{3^{-}}$and caffeine- gated stores in hippocampal and cortical neurons in $3 \times \mathrm{Tg}-\mathrm{AD}$ mice $[39,40,52]$. The gating of $\mathrm{IP}_{3} \mathrm{R}$ can be modulated by PS1-M146L and several other PS1-FAD mutants [53,54]. Xenopus laevis oocytes expressing PS1-M146V have increased sarco-/endoplasmic reticulum $\mathrm{Ca}^{2+}$ ATPase (SERCA) activity compared to those with wild-type PS1 [55], a mechanism that could contribute to the overfilling of ER $\mathrm{Ca}^{2+}$ store. Defects in store-operated $\mathrm{Ca}^{2+}$ entry have been reported in studies with PS1-FAD and PS knockout cells. Taken together, PSs have profound effects on the activity and/or expression of many proteins involved in intracellular $\mathrm{Ca}^{2+}$ signalling.

\section{Presenilins are ER $\mathrm{Ca}^{2+}$ leak channels}

Recently, we discovered that PSs function as low-conductance, passive ER $\mathrm{Ca}^{2+}$ leak channels, independent of $\gamma$-secretase activity [56]. We found that many FAD mutations in PS1 disrupt this $\mathrm{Ca}^{2+}$ leak function [56-58], resulting in overloaded $\mathrm{ER} \mathrm{Ca}^{2+}$ stores and exaggerated $\mathrm{ER} \mathrm{Ca}^{2+}$ 
release in double PS knock-out fibroblasts [56-58], cultured hippocampal neurons from PS double-knockout mice and PS1-M146V mutant neurons [51] and lymphoblasts from FAD patients [58]. These observations suggest that PS1 plays a pivotal role in deranged $\mathrm{Ca}^{2+}$ in $\mathrm{AD}$ and they also provide further support for the contribution of PS1 to $\mathrm{Ca}^{2+}$ homeostasis in neurons.

Our novel proposal that PSs function as $\mathrm{Ca}^{2+}$ or ion conductance channels has been challenged [53,54]. In particular, it has been argued that PSs lack an ion conductance pore and therefore would not be able to function as ion channels $[53,54]$. The crystal structure of PS has not been elucidated and the presence of an ion conductance pore cannot be confirmed or refuted based on structural analysis studies. However, recent biochemical studies of PS1 by two independent groups [59-62] have revealed the presence of a water-filled cavity within the hydrophobic membrane, which would allow for the hydrolysis required for proteolytic cleavage by $\gamma$-secretase. These groups utilized cysteine-scanning mutagenesis (SCAM), a technique that was developed to identify residues which line a pore of a channel $[63,64]$. In studies of PS1 it was determined that TM7 domain containing the catalytic aspartate residue D385 is facing a water-filled cavity in the lipid bilayer. In contrast, TM6 domain containing D257 catalytic residue was not water accessible. It was reasoned that significant translocation of TM6 is required to support proteolytic cleavage of the $\gamma$-secretase substrates within the membrane [59-62] (Figure 1B). Again using SCAM, the investigators were also able to demonstrate the involvement of TM domain 9 in forming the hydrophilic pore [60,61]. Being water-accessible and highly flexible, TM domain 9 is potentially involved in the translocation of substrates from the initial binding site to the catalytic site of PS1 [60]. Recent NMR studies of PS1-CTF in micelles revealed unusual features of TM domains 7 and 9, where TM domain 7 was discovered to be a putative half-membrane-spanning helix and TM domain 9 was a kinked helix [65]. In addition, they discovered a higher rate of hydrogen-deuterium exchange in these regions, implying either higher exposure to the aqueous environment and/or increased dynamics [65]. These data further confirm the flexibility of TM domain 9 and the involvement of TM domain 7 and 9 in forming a waterfilled catalytic core.

Could this hydrophilic pore (Figure 1B) also serve as an ion conductance channel? Recently, in order to answer this question and to map the ion conductance pore in PS1, we systematically evaluated ER $\mathrm{Ca}^{2+}$ leak activity supported by a series of cysteine point mutants in TM6, TM7 and TM9 of mPS1 expressed in PS double knockout mouse embryonic fibroblasts [66]. Using $\mathrm{Ca}^{2+}$ imaging and stable cell rescue lines, our results indicated that TM7 and TM9, but not TM6, play an important role in forming the ion/ $\mathrm{Ca}^{2+}$ conductance pore of PS1 [66]. These results are consistent with previous SCAM and NMR analysis of PS1 [59-62,65] and provide further support to our hypothesis that the hydrophilic catalytic cavity of presenilins also constitutes the $\mathrm{Ca}^{2+}$ conductance pore. However, it is important to remember that $\mathrm{Ca}^{2+}$ channel function is supported by the holoprotein of PS1 in the ER [56] and $\gamma$-secretase function is supported by cleaved PS1 in the plasma membrane [67]. Therefore, these 2 functions are mutually exclusive and never performed by the same PS1 molecule simultaneously (Figure 1B).

What about the location and composition of the ion $/ \mathrm{Ca}^{2+}$ conductance channel? Interestingly, it was discovered that PS1 forms homodimers in intact mammalian cells in fluorescent lifetime imaging microscopy using differentially labeled PS1 constructs experiments [68]. It has been reported that PS1 dimers affect $\gamma$-secretase activity and function [69-71]. However, the involvement of PS1 dimers and how the configuration relates to the ion conductance function of PS1 is not known. It was revealed that PS1 holoproteins with D257A mutation were also able to form dimers, suggesting that cleavage within the loop was not necessary for dimerization [68]. These data align with our finding that residues in TM domain 6, which contains D257, did not affect the $\mathrm{Ca}^{2+}$ channel function of PS1 and could support a possible homodimer configuration of PS1 for the ion channel [66]. Further experiments utilizing mutations of other TM domains that could be involved in the formation of the ion $/ \mathrm{Ca}^{2+}$ conductance pore of PSs are required. For example, recent application of SCAM approach demonstrated that TM1 of mPS1 is water exposed and facing the catalytic pore of $\gamma$-secretase [72]. However, for definitive information regarding the location and composition of the PS1 ion $/ \mathrm{Ca}^{2+}$ conductance pore, the crystal structure of PS must be elucidated.

\section{$5 \quad \mathrm{ER} \mathrm{Ca}^{2+}$ leak and variant $\mathrm{AD}$}

Clinical studies indicated that many patients with FAD mutations in PS1 display significant phenotypic heterogeneity, including variable age of onset, penetrance, myoclonus and seizures, Parkinsonism, apraxia/ataxia, FTD and psychiatric symptoms [73-75]. There are also variations in PS1-FAD neuropathology. For instance, greater NFT formation, altered amyloid $\beta$ plaque composition, hippocampal sclerosis, the appearance of Pick bodies and neuropathological involvement of the basal ganglia and/or brainstem [73-75]. An intriguing subset of PS1-FAD patients manifest symptoms of spastic paraparesis (SP, known as variant AD), or progressive spasticity of the lower limbs [73-76]. The linkage of SP to PS1-FAD mutations, R278T and M233T, was first reported in 1997 [77]. The PS1 deletion of exon $9(\Delta \mathrm{E} 9)$ was associated with SP in a Finnish family [78]. However, not all patients carrying PS1- $\Delta$ E9 within the same family will present with SP [79], which suggests the involvement of modifiers and environmental factors. Upon pathological examination of patients harbouring PS1-FAD mutations 

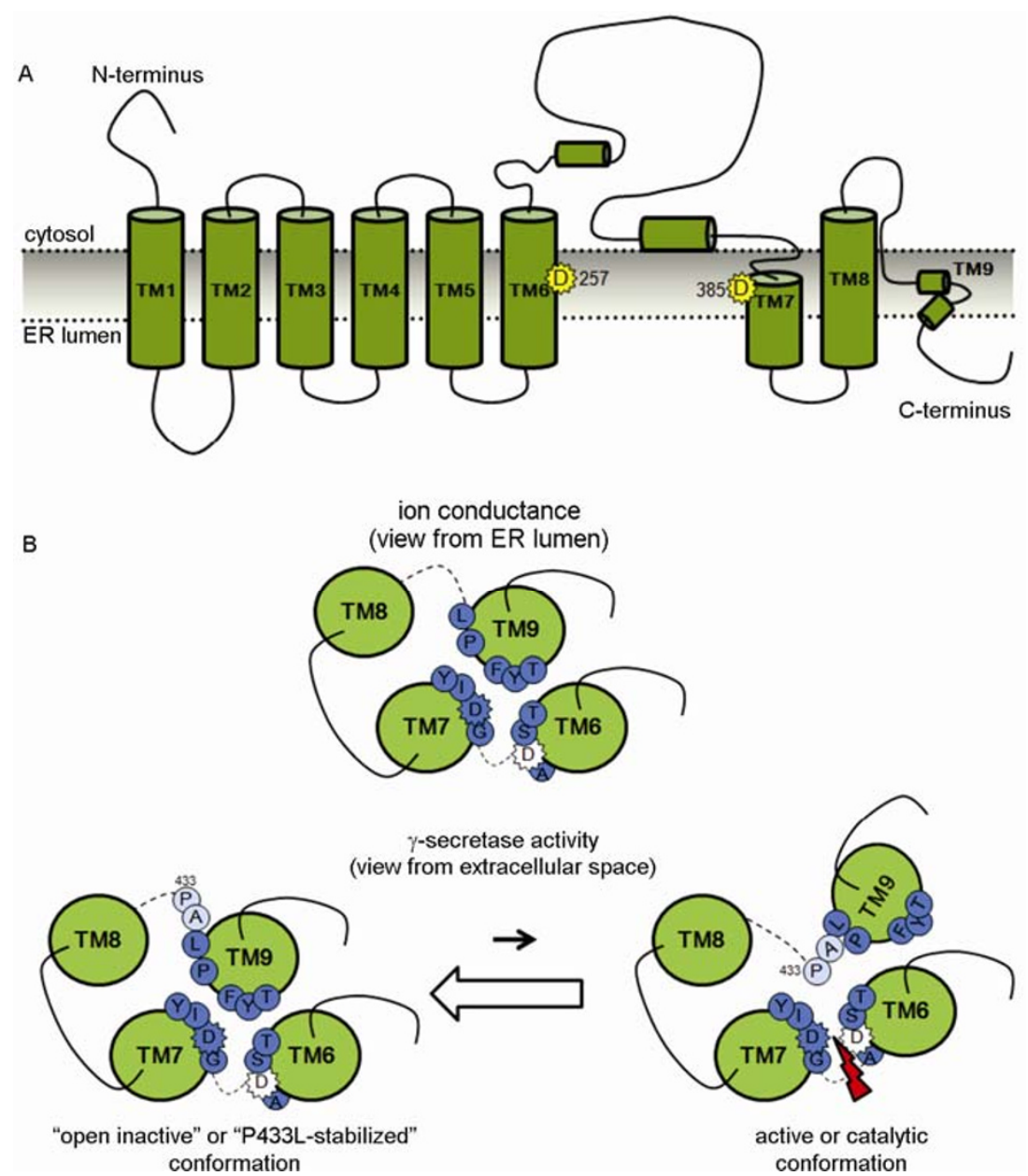

Figure $1 \mathrm{ER} \mathrm{Ca}^{2+}$ leak and $\gamma$-secretase function of PS1. A, The domain structure of PS1. TM domains 1-9 are indicated. The yellow stars indicate the catalytic asparates D257 and D385. Based on biochemical [17,18] and structural [65] studies. B, ER Ca ${ }^{2+}$ channel (top) and $\gamma$-secretase (bottom) conformations of hydrophilic pore in PS1. Adapted from [66]. Proposed arrangement of TM6, TM7, TM8 and TM9 is indicated. Amino acids are represented by a single letter code. Light blue residues are exposed to water in SCAM experiments and dispensible for $\mathrm{ER} \mathrm{Ca}^{2+}$ leak activity. Dark blue residues are exposed to water and essential for ER $\mathrm{Ca}^{2+}$ leak activity. Stars indicate the D257 and D385. The model is based on SCAM analysis [59-62] and results of ER Ca ${ }^{2+}$ leak assays [66].

associated with SP, their brains usually contain abundant large, non-cored amyloid- $\beta$ plaques, named cotton wool plaques (CWP) composed primarily of $\mathrm{A} \beta 42$ peptide and lacking surrounding neuritic dystrophy and glial activation $[8,73,80,81]$. Patients also display corticospinal tract degeneration at the level of the medulla and the spinal cord [79]. It is believed that the accumulation of CWP plaques in the basal ganglia, brainstem and spinal cord is responsible for the Parkinsonism and spastic phenotypes clinically observed in these patients. The reasons for these unique clinical and pathological phenotypes observed in these PS1-FAD pedigrees are not understood [8,73-75,80,81].

Could this variant AD somehow be explained by the altered ER $\mathrm{Ca}^{2+}$ signaling caused by PS1-FAD mutations? Over a series of experiments [56-58], we have tested a total of 23 FAD mutations in PS1, 1 FAD mutation in PS2 and 3 FTD implicated PS1 mutations. We concluded that 14 FAD mutations abolished ER $\mathrm{Ca}^{2+}$ leak function of PS (Figure 2A). These conclusions are based on the lack of channel activity in planar bilayers with recombinant PSs and by failure of these mutants to rescue $\mathrm{Ca}^{2+}$ signaling defects in PS DKO MEF cells. In contrast, another 10 FAD mutations in PS1 appeared to be functional and were able to rescue ER $\mathrm{Ca}^{2+}$ leak defects in PS DKO fibroblasts (Figure 2A). The PS1- $\triangle$ E9 FAD mutant acted as a "gain of function" (GOF) mutant based on bilayer experiments [56]. Other functional PS1 mutants were able to rescue $\mathrm{Ca}^{2+}$ signaling defects in PS DKO MEF cells but were not tested in bilayers. These mutants may correspond to a GOF, normal function, or partial loss of function mutations and were classified as "functional". All 3 PS1 mutants implicated in FTD (L113P,

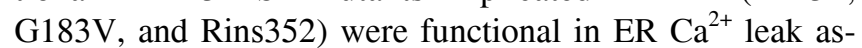
say (Figure 2A). The Rins352 mutation is probably not pathogenic as the same patient also has a mutation in progranulin, which is most likely responsible for the disease [13]. The pathogenic status of the L113P and G183V mutations remains to be elucidated. The "loss of function" (LOF) mutations are spread out through the sequence of PS1 


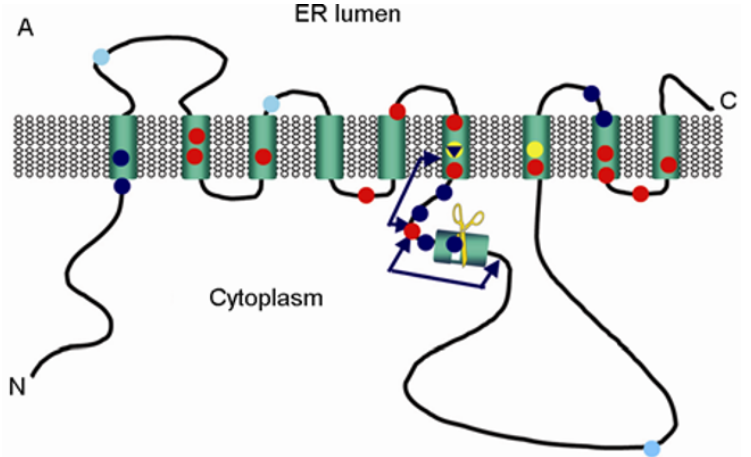

Loss of function(O)- M139V, M146VIM, L166P, G217D, K239E, A246E, V261F, E273A, G384A, L420R, A426P, A431E, P436Q; PS2-N141/L

Functional (-) - A79V, L85P, P264L, R269G, $\triangle E 8$, $\triangle$ EE9, E280G, T291P, N405S, C410Y FTD-implicated ( O)-L113P, G183V, Rins352

Y-Secretase catalytic Asp ( $)$ - D257, D385

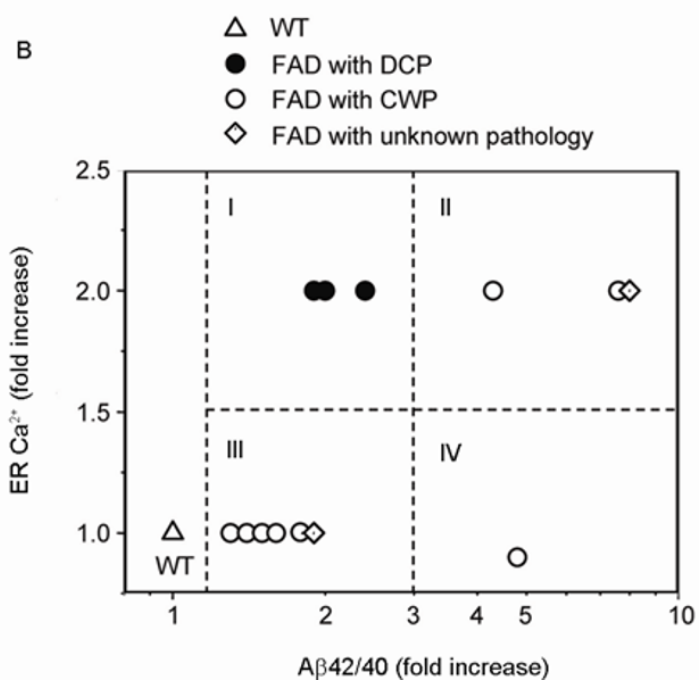

Figure 2 Variant $\mathrm{AD}$ and $\mathrm{ER} \mathrm{Ca}^{2+}$ leak function of PS1. A, Schematic representation of PS1-FAD mutations analyzed in $\mathrm{ER} \mathrm{Ca}^{2+}$ leak function experiments. Adapted from [58]. The nine transmembrane domains (TM1TM9) of presenilins, the $\gamma$-secretase catalytic aspartate residues (D257 and D385, yellow) and the site of the endoproteolytic cleavage of presenilins are shown. The effects of FAD mutations on ER $\mathrm{Ca}^{2+}$ leak activity of presenilins are color coded - red color is used for LOF mutations, blue color is used for "functional" mutations. The positions of 3 PS1 FTD-implicated mutants are also shown (light blue color). B, Effects of PS1-FAD mutations on $\mathrm{ER} \mathrm{Ca}^{2+}$ levels and $\mathrm{A} \beta 42 / 40$ ratios. Adapted from [58]. For each PS1-FAD mutant the $\mathrm{A} \beta 42 / 40$ ratios and $\mathrm{ER} \mathrm{Ca}^{2+}$ levels were normalized to the corresponding values for wild-type (WT) (open triangle). The PS1-FAD mutants with confirmed DCP pathology (M139V, M146L, A246E) are plotted as solid circles. The PS1-FAD mutants with confirmed CWP pathology (L166P, P436Q, P264L, $\Delta$ E8, E280G, $\Delta$ E9, T291P, $\mathrm{C} 410 \mathrm{Y})$ are plotted as open circles. The PS1-FAD mutants with unknown pathology (G384A, L85P) are plotted as open diamonds. The diagram is divided into 4 quadrants (I, II, III, IV) as shown and explained in the text.

(within exons 5, 6, 7, 8, 11, and 12 of PSEN1 gene) and are mostly localized to the TM domains (Figure 2A). Most "functional" PS1 FAD mutations are concentrated in the initial portion of a large cytosolic loop between TM6 and TM7 (Figure 2A), the region encoded by exons 8 and 9 of the PSEN1 gene.

Is there any correlation between $\mathrm{ER}^{\mathrm{Ca}^{2+}}$ leak phenotype of PS1-FAD mutants and clinical phenotypes of FAD patients with the same mutations? In our analysis we discovered that PS1- $\triangle \mathrm{E} 9$ and most other PS1-FAD mutants that were "functional" for $\mathrm{Ca}^{2+}$ leak (L85P, P264L, R269G, $\Delta E 8$, E280G, T291P, N405S, and C410Y) segregated with the CWP pathology and/or SP clinical phenotype. Remarkably, all "functional" mutations are associated with CWP/SP phenotype and none of the "functional" mutations led to typical dense-core plaque (DCP) AD pathology. Based on these results we concluded that the disruption of both ER $\mathrm{Ca}^{2+}$ leak and $\gamma$-secretase function of PS1 causes pathological changes in the brains of PS1-FAD patients. To represent these ideas quantitatively, we plotted the increase in $\mathrm{A} \beta 42 / 40$ ratio against the increase in $\mathrm{ER} \mathrm{Ca}^{2+}$ levels for each of the PS1-FAD mutants (Figure 2B). The fold increase values for $A \beta 42 / 40$ ratios (Figure $2 B$ ) for each of the PS1 FAD mutants were extracted from http://www.molgen. ua.ac.be/ADMutations [12,13]. The change in $\mathrm{ER} \mathrm{Ca}^{2+} \mathrm{lev-}$ els was plotted based on our studies of $\mathrm{ER} \mathrm{Ca}^{2+}$ leak activity. We made an assumption that for LOF mutants the ER $\mathrm{Ca}^{2+}$ levels were increased 2 fold, for "functional" mutants the $\mathrm{ER} \mathrm{Ca}^{2+}$ levels were unchanged, and for the GOF mutant PS1- $\triangle E 9$ the ER $\mathrm{Ca}^{2+}$ levels were reduced by $10 \%$. The $\mathrm{A} \beta 42 / 40$ ratios and $\mathrm{ER} \mathrm{Ca}^{2+}$ levels were normalized to the corresponding values for the wild-type (WT, open triangle). The 3 PS1-FAD mutants with confirmed DCP pathology (M139V, M146L, A246E) [82-84] were plotted as solid circles, the 8 PS1-FAD mutants with confirmed CWP pathology (L166P, P436Q, P264L, $\Delta$ E8, E280G, $\Delta$ E9, T291P, $\mathrm{C} 410 \mathrm{Y})[73,80,81]$ were plotted as open circles, and the 2 PS1-FAD mutants with unknown pathology (G384A, L85P) were plotted as diamonds. For the remaining 9 PS1-FAD mutants (G217D, K239E, V261F, E273A, L420R, A426P, A431E, R269G, and N405S) A $\beta 42 / 40$ values were not available and these mutants could not yet be plotted. The late onset, partially penetrant PS1-A79V mutation and PS2-N141I mutation were not plotted.

The resulting diagram can be divided into 4 quadrants (Figure 2B): quadrant I (high ER $\mathrm{Ca}^{2+}$, small increase in $\mathrm{A} \beta 42 / 40$ ), quadrant II (high ER $\mathrm{Ca}^{2+}$, large increase in $\mathrm{A} \beta 42 / 40$ ), quadrant III (low ER $\mathrm{Ca}^{2+}$, small increase in $\mathrm{A} \beta 42 / 40$ ), quadrant IV (low ER $\mathrm{Ca}^{2+}$, large increase in A $\beta 42 / 40)$. Interestingly, all 3 mutations with confirmed DCP are located in quadrant I, and all mutations with confirmed CWP are located in quadrants II, III, or IV (Figure 2B). Given these results, we propose that both $\mathrm{ER} \mathrm{Ca}^{2+}$ dyshomeostasis and increases in $A \beta 42 / 40$ ratios can play a role in development of $\mathrm{AD}$ pathology. If the pathology is driven by both $\mathrm{ER} \mathrm{Ca}^{2+}$ overload and an increase in $A \beta 42 / 40$ ratios, then the formation of DCP is favoured (quadrant I). However, if the pathology is primarily driven by an increase in the $A \beta 42 / 40$ ratio, then the formation of A 342 -enriched CWP plaques is strongly favored. We propose that this situation can occur when $\mathrm{ER} \mathrm{Ca}^{2+}$ levels are not affected (quadrant III) or when $\mathrm{ER} \mathrm{Ca}^{2+}$ levels are af- 
fected but an increase in $A \beta 42 / 40$ ratios is large (quadrant II). The extreme case of this situation is quadrant IV, which corresponds to both low ER $\mathrm{Ca}^{2+}$ levels and large increase in $A \beta 42 / 40$ ratios (Figure 2B). Indeed, the only mutation located in quadrant IV (PS1- $\triangle \mathrm{E} 9$ ) results in the most robust and penetrant CWP/SP clinical phenotype [85,86]. The proposed classification has a predictive power. For example, we predict that mutations G384A (located in quadrant II) and L85P (located in quadrant III) should also be linked with CWP pathology. It will be of interest to test this prediction experimentally. Although the pathological evaluation of these mutants has not yet been reported, the SP clinical phenotype has been previously described for PS1-L85P patients [87], consistent with our predictions.

The proposed hypothesis provides a novel insight into the relative contributions of $\mathrm{Ca}^{2+}$-driven and amyloiddriven pathological pathways in $\mathrm{AD}$ patients. Therefore, if pathology is primarily driven by an increase in A $\beta 42 / 40$ ratio, then formation of $\mathrm{A} \beta 42$-enriched CWP plaques is favored. It has been previously proposed that only mutations that cause large increases in $A \beta 42 / 40$ ratios, such as PS1- $\triangle E 9$ and PS1-436Q, can lead to CWP plaques [76]. Our proposal can be considered as expansion of the same idea which now adds " $\mathrm{Ca}^{2+}$ dimension" to the "amyloid dimension". In agreement with the earlier proposal [76], we argue that PS1-FAD mutations linked with large increases in $A \beta 42 / 40$ ratio should yield CWP phenotype independently from effects of the same mutation on ER $\mathrm{Ca}^{2+}$ leak (quadrants II and IV on Figure 2B). However, even PS1 mutations that have relatively small effects on $A \beta 42 / 40$ ratios would yield CWP phenotype if $\mathrm{ER} \mathrm{Ca}^{2+}$ leak is not effected (quadrant III on Figure 2B).

On the other hand, if pathology is driven by both $\mathrm{Ca}^{2+}$ dyshomeostasis and relatively modest increases in the A $342 / 40$ ratio, then typical DCP or "diffuse" plaques are favored (quadrant I on Figure 2B). It is possible that the relative contribution of these pathways may vary in different members of the same pedigree, resulting in a variable presentation of CWP/SP phenotype in most cases [73,80,81]. In agreement with this idea, the most robust CWP/SP phenotype was reported for PS1 $\triangle \mathrm{E} 9$ mutant $[78,85]$, which has the largest effect on $\gamma$-secretase activity and acts as a GOF mutant for $\mathrm{ER} \mathrm{Ca}^{2+}$ leak channels (quadrant IV, Figure 2B). Our hypothesis indeed predicts that the mutant with these properties should shift the balance as far as possible towards pure amyloid-driven pathology and formation of CWP plaques.

\section{Neuronal $\mathrm{Ca}^{2+}$ signalling and sporadic $\mathrm{AD}$}

What are the implications of these findings for sporadic AD? The age-related changes in neuronal $\mathrm{Ca}^{2+}$ signalling have been well established [88-90]. It has been shown that $\mathrm{Ca}^{2+}$ release from intracellular stores is increased in the aging neurons, similar to neurons that express PS1-FAD mutants with loss of $\mathrm{ER} \mathrm{Ca}^{2+}$ leak leak function. Thus, it is possible that overloaded ER $\mathrm{Ca}^{2+}$ stores result in "accelerated aging" phenotype [91]. Other features of aging neurons include depolarized mitochondria, reduced cytosolic $\mathrm{Ca} 2+$ buffering capacity, and activation of calcineurin and calpains. All these changes indicate that aging neurons experience significant dysregulation of intracellular $\mathrm{Ca}^{2+}$ handling. Consistent with importance of $\mathrm{Ca}^{2+}$ signalling in sporadic $\mathrm{AD}$, most sporadic $\mathrm{AD}$ patients present dense core plaque phenotype. However, some of sporadic AD patients also present CWP pathology [84], suggesting that CWP are not specific for early onset FAD resulting from PS1 mutations. The evidence suggests that there is a significant and complex interplay between the "amyloid hypothesis" and the " $\mathrm{Ca}^{2+}$ dysregulation hypothesis" of AD pathogenesis, and it appears that PSs are master-regulators of these processes. It is possible that the relative contribution of " $\mathrm{Ca}^{2+}$-driven" and "amyloid-driven" pathogenic processes may vary between different brain regions, resulting in different abundance of CWP and DCP plaques in different areas of the brain for SAD and FAD patients. Future studies will be needed to test the proposed hypothesis and to relate our findings to understanding the pathogenesis of familial and sporadic AD.

IB is a holder of Carl J. and Hortense M. Thomsen chair in Alzheimer's disease research and supported by the McKnight Neuroscience of Brain Disorders Award and NIH grant R01AG030746.

1 Brookmeyer R, Johnson E, Ziegler-Graham K, et al. Forecasting the global burden of Alzheimer's disease. Alzheimers Dement, 2007, 3: 186-191

2 Wenk G L. Neuropathologic changes in Alzheimer's disease. J Clin Psychiatry, 2003, 64 Suppl 9: 7-10

3 Giannakopoulos P, Kovari E, Gold G, et al. Pathological substrates of cognitive decline in Alzheimer's disease. Front Neurol Neurosci, 2009, 24: 20-29

4 Hardy J. The amyloid hypothesis for Alzheimer's disease: a critical reappraisal. J Neurochem, 2009, 110: 1129-1134

5 Hardy J, Selkoe D J. The amyloid hypothesis of Alzheimer's disease: progress and problems on the road to therapeutics. Science, 2002, 297: 353-356

6 Small S A, Duff K. Linking Abeta and tau in late-onset Alzheimer's disease: a dual pathway hypothesis. Neuron, 2008, 60: 534-542

7 Bertram L, Tanzi R E. Thirty years of Alzheimer's disease genetics: the implications of systematic meta-analyses. Nat Rev Neurosci, 2008, 9: 768-778

8 Bergmans B A, De Strooper B. gamma-secretases: from cell biology to therapeutic strategies. Lancet Neurol, 2010, 9: 215-226

9 Hutton M, Lendon C L, Rizzu P, et al. Association of missense and 5'-splice-site mutations in tau with the inherited dementia FTDP-17. Nature, 1998, 393: 702-705

10 Cruts M, Van Broeckhoven C. Loss of progranulin function in frontotemporal lobar degeneration. Trends Genet, 2008, 24: 186-194

11 Sherrington R, Rogaev E I, Liang Y, et al. Cloning of a gene bearing missense mutations in early-onset familial Alzheimer's disease. Nature, 1995, 375: 754-760

12 Cruts M, Brouwers N. Alzheimer disease \& frontotemporal dementia mutation database. Available at: www.molgen.ua.ac.be/Admutations

13 Cruts M, Van Broeckhoven C. Presenilin mutations in Alzheimer's disease. Hum Mutat, 1998, 11: 183-190 
14 Urban S, Schlieper D, Freeman M. Conservation of intramembrane proteolytic activity and substrate specificity in prokaryotic and eukaryotic rhomboids. Curr Biol, 2002, 12: 1507-1512

15 Brown M S, Ye J, Rawson R B, et al. Regulated intramembrane proteolysis: a control mechanism conserved from bacteria to humans. Cell, 2000, 100: 391-398

16 Hass M R, Sato C, Kopan R, et al. Presenilin: RIP and beyond. Semin Cell Dev Biol, 2009, 20: 201-210

17 Laudon H, Hansson E M, Melen K, et al. A nine-transmembrane domain topology for presenilin 1. J Biol Chem, 2005, 280: 3535235360

18 Spasic D, Tolia A, Dillen K, et al. Presenilin-1 maintains a ninetransmembrane topology throughout the secretory pathway. J Biol Chem, 2006, 281: 26569-26577

19 Annaert W G, Levesque L, Craessaerts K, et al. Presenilin 1 controls gamma-secretase processing of amyloid precursor protein in pregolgi compartments of hippocampal neurons. J Cell Biol, 1999, 147: 277-294

20 Thinakaran G, Borchelt D R, Lee M K, et al. Endoproteolysis of presenilin 1 and accumulation of processed derivatives in vivo. Neuron, 1996, 17: 181-190

21 De Strooper B, Beullens M, Contreras B, et al. Phosphorylation, subcellular localization, and membrane orientation of the Alzheimer's disease-associated presenilins. J Biol Chem, 1997, 272: 3590-3598

$22 \mathrm{Yu} \mathrm{G}$, Chen F, Levesque G, et al. The presenilin 1 protein is a component of a high molecular weight intracellular complex that contains beta-catenin. J Biol Chem, 1998, 273: 16470-16475

23 Kimberly W T, LaVoie M J, Ostaszewski B L, et al. Gamma-secretase is a membrane protein complex comprised of presenilin, nicastrin, Aph-1, and Pen-2. Proc Natl Acad Sci USA, 2003, 100: 6382-6387

24 Edbauer D, Winkler E, Regula J T, et al. Reconstitution of gamma-secretase activity. Nat Cell Biol, 2003, 5: 486-488

25 Takasugi N, Tomita $\mathrm{T}$, Hayashi I, et al. The role of presenilin cofactors in the gamma-secretase complex. Nature, 2003, 422: $438-441$

26 Lazarov V K, Fraering $\mathrm{P}$ C, Ye W, et al. Electron microscopic structure of purified, active gamma-secretase reveals an aqueous intramembrane chamber and two pores. Proc Natl Acad Sci USA, 2006, 103: 6889-6894

27 Wolfe M S, Xia W, Ostaszewski B L, et al. Two transmembrane aspartates in presenilin-1 required for presenilin endoproteolysis and gamma-secretase activity. Nature, 1999, 398: 513-517

28 De Strooper B, Saftig P, Craessaerts K, et al. Deficiency of presenilin-1 inhibits the normal cleavage of amyloid precursor protein. Nature, 1998, 391: 387-390

29 Scheuner D, Eckman C, Jensen M, et al. Secreted amyloid beta-protein similar to that in the senile plaques of Alzheimer's disease is increased in vivo by the presenilin 1 and 2 and APP mutations linked to familial Alzheimer's disease. Nat Med, 1996, 2: $864-870$

30 Supnet C, Bezprozvanny I. The dysregulation of intracellular calcium in Alzheimer disease. Cell Calcium, 2010, 47: 183-189

31 Bezprozvanny I, Mattson M P. Neuronal calcium mishandling and the pathogenesis of Alzheimer's disease. Trends Neurosci, 2008, 31: 454-463

32 Stutzmann G E. The pathogenesis of Alzheimers disease is it a lifelong "calciumopathy"? Neuroscientist, 2007, 13: 546-559

33 Demuro A, Parker I, Stutzmann G E. Calcium signaling and amyloid toxicity in Alzheimer disease. J Biol Chem, 2010, 285: 12463-12468

34 Ito $\mathrm{E}$, Oka $\mathrm{K}$, Etcheberrigaray $\mathrm{R}$, et al. Internal $\mathrm{Ca}^{2+}$ mobilization is altered in fibroblasts from patients with Alzheimer disease. Proc Natl Acad Sci USA, 1994, 91: 534-538

35 Etcheberrigaray R, Hirashima N, Nee L, et al. Calcium responses in fibroblasts from asymptomatic members of Alzheimer's disease families. Neurobiol Dis, 1998, 5: 37-45

36 Khachaturian Z S. Calcium, membranes, aging, and Alzheimer's disease. Introduction and overview. Ann N Y Acad Sci, 1989, 568: $1-4$
37 Supnet C, Bezprozvanny I. Neuronal calcium signaling, mitochondrial dysfunction, and Alzheimer's disease. J Alzheimers Dis, 2010, 20 Suppl 2: S487-S498

38 Stutzmann G E, Caccamo A, LaFerla F M, et al. Dysregulated IP3 signaling in cortical neurons of knock-in mice expressing an Alzheimer's-linked mutation in presenilin1 results in exaggerated $\mathrm{Ca}^{2+}$ signals and altered membrane excitability. J Neurosci, 2004, 24 : 508-513

39 Stutzmann G E, Smith I, Caccamo A, et al. Enhanced ryanodine receptor recruitment contributes to $\mathrm{Ca}^{2+}$ disruptions in young, adult, and aged Alzheimer's disease mice. J Neurosci, 2006, 26: 5180-5189

40 Chakroborty S, Goussakov I, Miller M B, et al. Deviant ryanodine receptor-mediated calcium release resets synaptic homeostasis in presymptomatic 3×Tg-AD mice. J Neurosci, 2009, 29: 9458-9470

41 Smith I F, Hitt B, Green K N, et al. Enhanced caffeine-induced $\mathrm{Ca}^{2+}$ release in the $3 \times \mathrm{Tg}-\mathrm{AD}$ mouse model of Alzheimer's disease. J Neurochem, 2005, 94: 1711-1718

42 Chan S L, Mayne M, Holden C P, et al. Presenilin-1 mutations increase levels of ryanodine receptors and calcium release in $\mathrm{PC} 12$ cells and cortical neurons. J Biol Chem, 2000, 275: 18195-18200

43 Lee S Y, Hwang D Y, Kim Y K, et al. PS2 mutation increases neuronal cell vulnerability to neurotoxicants through activation of caspase- 3 by enhancing of ryanodine receptor-mediated calcium release. FASEB J, 2006, 20: 151-153

44 Leissring M A, Parker I, LaFerla F M. Presenilin-2 mutations modulate amplitude and kinetics of inositol 1,4,5-trisphosphatemediated calcium signals. J Biol Chem, 1999, 274: 32535-32538

45 Leissring M A, Akbari Y, Fanger C M, et al. Capacitative calcium entry deficits and elevated luminal calcium content in mutant presenilin-1 knockin mice. J Cell Biol, 2000, 149: 793-798

46 Zatti G, Ghidoni R, Barbiero L, et al. The presenilin 2 M239I mutation associated with familial Alzheimer's disease reduces $\mathrm{Ca}^{2+}$ release from intracellular stores. Neurobiol of Dis, 2004, 15: 269-278

47 Giacomello M, Barbiero L, Zatti G, et al. Reduction of $\mathrm{Ca}^{2+}$ stores and capacitative $\mathrm{Ca}^{2+}$ entry is associated with the familial Alzheimer's disease presenilin-2 T122R mutation and anticipates the onset of dementia. Neurobiol Dis, 2005, 18: 638-648

48 Yoo A S, Cheng I, Chung S, et al. Presenilin-mediated modulation of capacitative calcium entry. Neuron, 2000, 27: 561-572

49 Herms J, Schneider I, Dewachter I, et al. Capacitive calcium entry is directly attenuated by mutant presenilin-1, independent of the expression of the amyloid precursor protein. J Biol Chem, 2003, 278: 2484-2489

50 Akbari Y, Hitt B D, Murphy M P, et al. Presenilin regulates capacitative calcium entry dependently and independently of gamma-secretase activity. Biochem Biophys Res Commun, 2004, 322: 1145-1152

51 Zhang H, Sun S, Herreman A, et al. Role of presenilins in neuronal calcium homeostasis. J Neurosci, 2010, 30: 8566-8580

52 Goussakov I, Miller M B, Stutzmann G E. NMDA-mediated $\mathrm{Ca}^{2+}$ influx drives aberrant ryanodine receptor activation in dendrites of young Alzheimer's disease mice. J Neurosci, 2010, 30: 12128-12137

53 Cheung K H, Shineman D, Muller M, et al. Mechanism of $\mathrm{Ca}^{2+}$ disruption in Alzheimer's disease by presenilin regulation of $\operatorname{Ins} \mathrm{P}(3)$ receptor channel gating. Neuron, 2008, 58: 871-883

54 Cheung K H, Mei L, Mak D O, et al. Gain-of-function enhancement of IP3 receptor modal gating by familial Alzheimer's disease-linked presenilin mutants in human cells and mouse neurons. Sci Signal, 2010, 3: ra22

55 Green K N, Demuro A, Akbari Y, et al. SERCA pump activity is physiologically regulated by presenilin and regulates amyloid beta production. J Cell Biol, 2008, 181: 1107-1116

56 Tu H, Nelson O, Bezprozvanny A, et al. Presenilins form ER calcium leak channels, a function disrupted by mutations linked to familial Alzheimer's disease. Cell, 2006, 126: 981-993

57 Nelson O, Tu H, Lei T, et al. Familial Alzheimer disease-linked mutations specifically disrupt $\mathrm{Ca}^{2+}$ leak function of presenilin 1 . J Clin Invest, 2007, 117: 1230-1239

58 Nelson O, Supnet C, Liu H, et al. Familial Alzheimer's disease 
mutations in presenilins: effects on endoplasmic reticulum calcium homeostasis and correlation with clinical phenotypes. J Alzheimers Dis, 2010, 21: 781-793

59 Tolia A, Chavez-Gutierrez L, De Strooper B. Contribution of presenilin transmembrane domains 6 and 7 to a water-containing cavity in the \{gamma\}-secretase complex. J Biol Chem, 2006, 281: 27633-27642

60 Tolia A, Horre K, De Strooper B. Transmembrane domain 9 of presenilin determines the dynamic conformation of the catalytic site of gamma-secretase. J Biol Chem, 2008, 283: 19793-19803

61 Sato C, Takagi S, Tomita T, et al. The C-terminal PAL motif and transmembrane domain 9 of presenilin 1 are involved in the formation of the catalytic pore of the gamma-secretase. J Neurosci, 2008, 28: 6264-6271

62 Sato C, Morohashi Y, Tomita T, et al. Structure of the catalytic pore of gamma-secretase probed by the accessibility of substituted cysteines. J Neurosci, 2006, 26: 12081-12088

63 Akabas M H, Stauffer D A, Xu M, et al. Acetylcholine receptor channel structure probed in cysteine-substitution mutants. Science, 1992, 258: 307-310

64 Akabas M H, Kaufmann C, Archdeacon P, et al. Identification of acetylcholine receptor channel-lining residues in the entire M2 segment of the alpha subunit. Neuron, 1994, 13: 919-927

65 Sobhanifar S, Schneider B, Lohr F, et al. Structural investigation of the C-terminal catalytic fragment of presenilin 1. Proc Natl Acad Sci USA, 2010, 107: 9644-9649

66 Nelson O, Supnet C, Tolia A, et al. Mutagenesis mapping of the presenilin 1 calcium leak conductance pore. J Biol Chem, 2011, 286: 22339-22347

67 De Strooper B. Aph-1, Pen-2, and Nicastrin with Presenilin generate an active gamma-secretase complex. Neuron, 2003, 38: 9-12

68 Herl L, Lleo A, Thomas A V, et al. Detection of presenilin-1 homodimer formation in intact cells using fluorescent lifetime imaging microscopy. Biochem Biophys Res Commun, 2006, 340: $668-674$

69 Cervantes S, Gonzalez-Duarte R, Marfany G. Homodimerization of presenilin $\mathrm{N}$-terminal fragments is affected by mutations linked to Alzheimer's disease. FEBS Lett, 2001, 505: 81-86

70 Hebert S S, Godin C, Tomiyama T, et al. Dimerization of presenilin1 in vivo: suggestion of novel regulatory mechanisms leading to higher order complexes. Biochem Biophys Res Commun, 2003, 301: 119-126

71 Cervantes S, Saura C A, Pomares E, et al. Functional implications of the presenilin dimerization: reconstitution of gamma-secretase activity by assembly of a catalytic site at the dimer interface of two catalytically inactive presenilins. J Biol Chem, 2004, 279: 3651936529

72 Takagi S, Tominaga A, Sato C, et al. Participation of transmembrane domain 1 of presenilin 1 in the catalytic pore structure of the \{gamma\}-secretase. J Neurosci, 2010, 30: 15943-15950

73 Larner A J, Doran M. Clinical phenotypic heterogeneity of Alzheimer's disease associated with mutations of the presenilin-1 gene. J Neurol, 2006, 253: 139-158

74 Ryan N S, Rossor M N. Correlating familial Alzheimer's disease gene mutations with clinical phenotype. Biomark Med, 2010, 4: 99-112

75 Shepherd C, McCann H, Halliday G M. Variations in the neuropathology of familial Alzheimer's disease. Acta Neuropathol, 2009, 118: 37-52

76 Houlden H, Baker M, McGowan E, et al. Variant Alzheimer's disease with spastic paraparesis and cotton wool plaques is caused by PS-1 mutations that lead to exceptionally high amyloid-beta concentrations. Ann Neurol, 2000, 48: 806-808

77 Kwok J B, Taddei K, Hallupp M, et al. Two novel (M233T and R278T) presenilin-1 mutations in early-onset Alzheimer's disease pedigrees and preliminary evidence for association of presenilin-1 mutations with a novel phenotype. Neuroreport, 1997, 8: 1537-1542

78 Crook R, Verkkoniemi A, Perez-Tur J, et al. A variant of Alzheimer's disease with spastic paraparesis and unusual plaques due to deletion of exon 9 of presenilin 1. Nat Med, 1998, 4: 452-455

79 Verkkoniemi A, Kalimo H, Paetau A, et al. Variant Alzheimer disease with spastic paraparesis: neuropathological phenotype. J Neuropathol Exp Neurol, 2001, 60: 483-492

80 Karlstrom H, Brooks W S, Kwok J B, et al. Variable phenotype of Alzheimer's disease with spastic paraparesis. J Neurochem, 2008, 104: 573-83

81 Tabira T, Chui D H, Nakayama H, et al. Alzheimer's disease with spastic paresis and cotton wool type plaques. J Neurosci Res, 2002, 70: $367-372$

82 Ataka S, Tomiyama $\mathrm{T}$, Takuma $\mathrm{H}$, et al. A novel presenilin-1 mutation (Leu85Pro) in early-onset Alzheimer disease with spastic paraparesis. Arch Neurol, 2004, 61: 1773-1776

83 Halliday G M, Song Y J, Lepar G, et al. Pick bodies in a family with presenilin-1 Alzheimer's disease. Ann Neurol, 2005, 57: 139-143

84 Le T V, Crook R, Hardy J, et al. Cotton wool plaques in non-familial late-onset Alzheimer disease. J Neuropathol Exp Neurol, 2001, 60: 1051-1061

85 Brooks W S, Kwok J B, Kril J J, et al. Alzheimer's disease with spastic paraparesis and 'cotton wool' plaques: two pedigrees with PS-1 exon 9 deletions. Brain, 2003, 126: 783-791

86 Jacquemont M L, Campion D, Hahn V, et al. Spastic paraparesis and atypical dementia caused by PSEN1 mutation (P264L), responsible for Alzheimer's disease. J Med Genet, 2002, 39: E2

87 Takao M, Ghetti B, Hayakawa I, Ikeda E, et al. A novel mutation (G217D) in the Presenilin 1 gene ( PSEN1) in a Japanese family: presenile dementia and parkinsonism are associated with cotton wool plaques in the cortex and striatum. Acta Neuropathol, 2002, 104: $155-170$

88 Toescu E C, Verkhratsky A. The importance of being subtle: small changes in calcium homeostasis control cognitive decline in normal aging. Aging Cell, 2007, 6: 267-273

89 Gant J C, Sama M M, Landfield P W, et al. Early and simultaneous emergence of multiple hippocampal biomarkers of aging is mediated by $\mathrm{Ca}^{2+}$-induced $\mathrm{Ca}^{2+}$ release. J Neurosci, 2006, 26: 3482-3490

90 Foster T C. Calcium homeostasis and modulation of synaptic plasticity in the aged brain. Aging Cell, 2007, 6: 319-325

91 Bezprozvanny I. Calcium signaling and neurodegenerative diseases. Trends Mol Med, 2009, 15: 89-100

Open Access This article is distributed under the terms of the Creative Commons Attribution License which permits any use, distribution, and reproduction in any medium, provided the original author(s) and source are credited. 